\title{
Change in refraction, corneal power and axial length of the eye secondary to silicone oil tamponade injection after pars plana vitrectomy
}

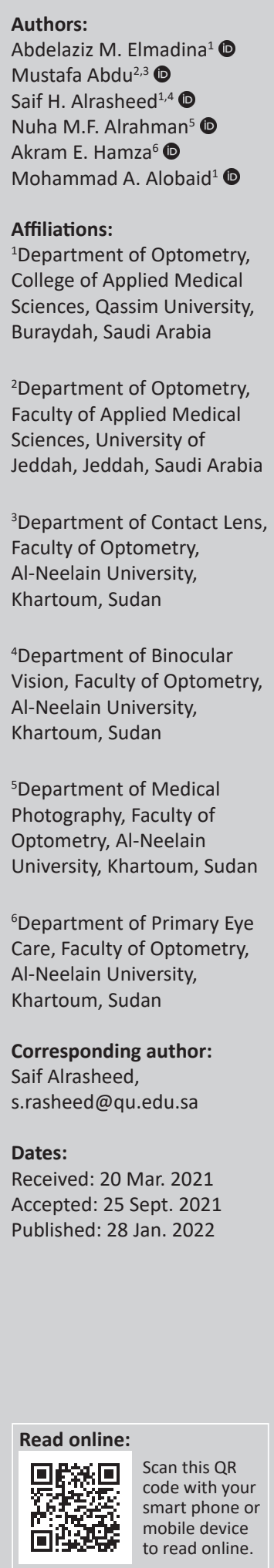

Background: Refractive and ocular biometric changes after silicone oil tamponade (SOT) in phakic eyes have been studied thoroughly; however, similar studies amongst Sudanese patients with vitreoretinal conditions were not carried out.

Aim: This study aimed to assess post-operative changes in refraction, corneal power and axial length after pars plana vitrectomy (PPV) with SOT in phakic patients.

Setting: The study was conducted at Sudan Eye Center, Khartoum, Sudan.

Methods: A cross-sectional retrospective hospital-based study was performed at Sudan Eye Center. The study included all phakic patients who underwent PPV with SOT for a variety of vitreoretinal conditions from October 2018 to January 2019. Clinical investigations included uncorrected visual acuity (VA), best-corrected VA, refraction, corneal power and axial length pre- and post-surgery. Differences in these parameters were assessed using a paired sample $t$-test with a significance level of $<0.05$.

Results: Medical records of 59 consecutive patients (59 eyes, 41 men and 18 women) with ages ranging from 22 years to 75 years were studied. The findings revealed a statistically significant hypermetropic change (pre: $-0.17 \pm 2.44$ dioptre [D] and post: $+4.43 \pm 2.04 \mathrm{D}$ ) with a $p$-value of $<0.001$. In terms of axial length, there was a significant increase (pre: $23.61 \pm 1.17 \mathrm{~mm}$ and post: $23.82 \pm 1.27 \mathrm{~mm} ; p<0.001)$. However, no significant change was found in corneal power (pre: $-43.60 \pm 1.96 \mathrm{D}$ and post: $43.63 \pm 2.03 \mathrm{D} ; p=0.45$ ).

Conclusion: A hypermetropic shift in refraction, longer axial length and non-considerable increase in corneal power were observed amongst Sudanese phakic patients after PPV with instillation of silicone oil.

Keywords: refractive status; Silicone Oil Tamponade; Pars Plana Vitrectomy; K-readings; axial length.

\section{Introduction}

The silicone oil (SO) is an effective intraocular tamponade material used in complex vitreoretinal surgeries with a higher refractive index (1.405) in comparison with the vitreous and aqueous humour. ${ }^{1,2}$ This oil when injected into the vitreous cavity produces a noticeable alteration in the refractive state of the eye. ${ }^{2,3}$ The use of $\mathrm{SO}$ as a long-acting retinal tamponade was found to increase the chances of keeping the retina in its position and improving the management of complicated vitreoretinal diseases. ${ }^{3}$ Silicone oil has a refractive index slightly higher than that of vitreous, and hence, it causes refractive changes, which are determined by the shape of the anterior surface of the silicone bubble and the positioning of the patient. ${ }^{3,4}$ A negative lens effect occurs in the phakic eyes because of the concavity produced by the posterior lens surface. Consequently, the eye becomes more hyperopic. ${ }^{4}$ Aphakic eyes experience a myopic shift because of the convexity of the anterior bubble surface. In the supine position, the oil separates from the retina producing a positive posterior surface contributing to myopia, where more effect occurs in aphakic eyes. ${ }^{3,4,5,6}$

Pavlović et al. $^{7}$ in a retrospective study reported that the mean difference in refraction after SO tamponade (SOT) in phakic eyes was +5.07 dioptres (Ds). In another study conducted by Fang et al., ${ }^{8}$ they found a significant myopic shift with the mean spherical equivalent of $-3.90 \pm 1.75 \mathrm{Ds}$

How to cite this article: Elmadina AM, Abdu M, Alrasheed SH, Alrahman NFM, Hamza AE, Alobaid MA. Change in refraction, corneal power and axial length of the eye secondary to silicone oil tamponade injection after pars plana vitrectomy. Afr Vision Eye Health. 2022;81(1), a667. https://doi.org/10.4102/aveh.v81i1.667

Copyright: @ 2022. The Author(s). Licensee: AOSIS. This work is licensed under the Creative Commons Attribution License. 
with SOT in pseudophakic eyes; however, Song et al. ${ }^{9}$ reported a mean spherical equivalent of $3.85 \pm 1.63 \mathrm{Ds}$, and after the removal of $\mathrm{SO}$, a myopic shift of $-4.51 \pm 1.79$ Ds was observed. Hoffman et al. ${ }^{10}$ concluded that:

$[H]$ yperopia was observed in all dogs that underwent silicon oil injection tamponade, regardless of lens status (phakic, pseudophakic, or aphakic). Pseudophakic eyes appeared to be more hyperopic than phakic eyes when filled with silicon oil. Based on Gullstrand schematic eye there is an increase of $8 \mathrm{D}$ hyperopia. (p. 1299)

In aphakic eyes, the SO produces a convex surface, thus creating a myopic refractive change the amount of myopic shift depends on the papillary aperture diameter. Based on the schematic eye calculation, a refractive shift from $+12.5 \mathrm{D}$ to $+5.6 \mathrm{D}$ has been observed in aphakic eyes filled with SO. ${ }^{11,12}$ Therefore, for all mentioned above, the current study was performed to further assess post-operative changes in refraction, corneal power and axial length after Pars Plana Vitrectomy (PPV) with SOT in phakic patients.

\section{Methods \\ Study design}

A retrospective cross-sectional hospital-based study was conducted in Sudan Eye Center, Khartoum, Sudan. The study included all patients who underwent PPV with SOT for a variety of vitreoretinal conditions from October 2018 to January 2019.

\section{Inclusion criteria}

Subjects included in this study had undergone PPV without scleral buckling, SOT agent. Fifty-nine consecutive patients (59 eyes, 41 men, and 18 women) with ages ranging from 22 years to 75 years were recruited.

\section{Data collection procedures}

Inner and outer eye examinations were performed by ophthalmologist and optometrist with significant expertise. Data collected included demographic characteristics of patients (age, gender, laterality [operated eye right or left]) and eye examinations, which include uncorrected visual acuity (VA), objective and subjective refraction, bestcorrected VA, keratometry readings and axial length of the eye. The above-mentioned examinations were performed pre- and post-surgery. The surgical procedures included the standard three-port pars plana techniques, which were carried out by one retinal consultant, , and SO injection was used in surgery. Screen Save Apramed VA test chart (E) was used to measure uncorrected VA and best-corrected VA, which was recorded in Snellen's decimal notation. Refraction and keratometry were performed using SHINNIPPON (Accuref k-900) auto kerato-refractometer; however, the axial length of the eye was measured using TOMEY Ultrasonic A/B Scanner (UD-8000).

\section{Data analysis}

Statistical analysis was performed using Statistical Package for the Social Sciences (SPSS) Windows version 21.0 (SPSS Inc., Chicago, IL, United States). Data were reported as means \pm standard deviations (s.d.). A paired sample $t$-test was performed to compare variables pre- and post-surgery. Furthermore, correlation analysis was conducted to assess the role of axial length and corneal curvature in refractive change before and after PPV. A $p$-value of $<0.05$ was considered to be statistically significant.

\section{Ethical considerations}

Ethical approval was obtained from Al-Neelain University, Khartoum, Sudan, and the study was performed according to the Declaration of Helsinki on the conduct of human research. Informed consent was waived because of the retrospective nature of the study. However, efforts were made to ensure that patients' confidentiality was guaranteed.

\section{Results \\ Demographic and clinical data}

The study included 59 participants (59 phakic eyes) who underwent PPV combined with SOT for different vitreoretinal conditions in one eye. Amongst them 41 (69.5\%) were men and $18(30.5 \%)$ were women with a mean age of $52.19 \pm 11.74$ years (range: $22-75$ years). In terms of refractive error, $40.7 \%$ of the patients were hypermetropic, 33.9\% myopic and $25.4 \%$ with faint reflex at baseline (before surgery). The mean uncorrected VA was $0.13 \pm 0.17$, whilst the mean bestcorrected VA was $0.17 \pm 0.20$. At baseline, means of refraction, average keratometry reading and axial length of the eye were $-0.17 \pm 2.44 \mathrm{D}, 43.60 \pm 1.96 \mathrm{D}$ and $23.61 \pm 1.17 \mathrm{~mm}$, respectively, as shown in Table 1.

\section{Inferential statistics}

Before starting this part of the analysis, assumptions for each statistical test were checked and found not violated. The findings revealed a statistically significanthypermetropic change (pre: $-0.17 \pm 2.44 \mathrm{D}$ and post: $+4.43 \pm 2.04 \mathrm{D}$ ) with a $p$-value of $<0.00$, as shown in Figure 2. In terms of axial length, there was a significant increase (pre: $-23.61 \pm 1.17$ $\mathrm{mm}$ and post: $23.82 \pm 1.27 \mathrm{~mm}$; $p<0.001)$. However, no significant change was found in corneal power (pre: 43.60 $\pm 1.96 \mathrm{D}$ and post: $43.63 \pm 2.03 \mathrm{D} ; p=0.45)$. There was a significant reduction in uncorrected VA (pre: $-0.13 \pm 0.17$ and: post $0.08 \pm 0.07 ; p=0.007)$. Conversely, there was a significant improvement in the best-corrected VA (pre: $-0.17 \pm 0.20$ and post: $0.24 \pm 0.22 ; p=0.01$ ), as shown in Table 2.

Further analysis showed negative correlations between refraction and axial length of the eye pre- and post-surgery with $p$-values of $<0.001$ and 0.04 , respectively. However, no significant correlations were detected between refraction and 
average corneal power in both measurements pre- and post-surgery (see Figure 1a, b).

\section{Discussion}

Currently, SO plays an important role in vitreoretinal surgery and is considered to be the material of choice as a long-term and stable retinal tamponade. The use of $\mathrm{SO}$ for vitreoretinal surgeries has many arguments with respect to its safety. Hence, the removal of SO is normally recommended. ${ }^{11,12,13,14}$ Prolonged SOT has been found to induce various eye complications, including the development of cataracts, glaucoma, keratopathy and change in refractive errors. ${ }^{14,15}$ Several factors may account for the refractive change after PPV with SOT in phakic patients, which include changes in cornea curvature, anterior chamber depth (ACD) and axial length of the eye. Conversely, the injection of $\mathrm{SO}$ into the vitreous cavity was found to produce a considerable hyperopic change in the refractive state, which could be because of the higher refractive index of the oil compared with the vitreous humor refractive index. ${ }^{16}$ These changes vary according to the lens state and the part of the vitreous cavity occupied by the SO; for this reason, SOT causes a hyperopic shift in phakic eyes. ${ }^{16,17,18}$ A study conducted by Gopal ${ }^{11}$ and Lakshmipriya showed 'that in a normal phakic eye, the silicone oil forms a concave surface behind the lens, thereby acting as a minus lens inside the eye making the eye hyperopic'. This might be explaining the hypermetropic change after SOT in PPV. Consequently, the current study was conducted to assess post-operative changes in refraction, corneal power and axial length because of PPV with SOT in phakic eyes.

An overall hypermetropic shift of $4.60 \mathrm{D}$ in refraction was observed in this study, which was statistically significant, $P<0.001$. The degree of refractive change was found to vary from $+0.50 \mathrm{D}$ to $+9.25 \mathrm{D}$. Similar findings were also reported in

TABLE 2: Pre-operative and post-operative comparison of variables.

\begin{tabular}{|c|c|c|c|}
\hline \multirow[t]{2}{*}{ Variable $(N=59)$} & \multicolumn{2}{|c|}{ Mean \pm s.d. } & \multirow[t]{2}{*}{$p$} \\
\hline & Pre-operative & Post-operative & \\
\hline Refraction & $-0.17 \pm 2.44$ & $4.43 \pm 2.04$ & $<0.001$ \\
\hline Corneal power & $43.60 \pm 1.96$ & $43.63 \pm 2.03$ & 0.450 \\
\hline Axial length & $23.61 \pm 1.17$ & $23.82 \pm 1.27$ & $<0.001$ \\
\hline Uncorrected VA & $0.13 \pm 0.17$ & $0.08 \pm 0.07$ & 0.007 \\
\hline Best-corrected VA & $0.17 \pm 0.20$ & $0.24 \pm 0.22$ & 0.010 \\
\hline
\end{tabular}

VA, visual acuity; s.d., standard deviation.

TABLE 1: Demographic and clinical characteristics of patients.

\begin{tabular}{|c|c|c|c|c|c|c|}
\hline Characteristic (59 patients, 59 eyes) & Frequency & $\%$ & Mean \pm s.d. & Range & Mean & Dioptre \\
\hline Age & - & - & $52.19 \pm 11.74$ & - & - & - \\
\hline Range (years) & - & - & - & $22-75$ & - & - \\
\hline Gender & - & - & - & - & - & - \\
\hline Male & 41 & 69.5 & - & - & - & - \\
\hline Female & 18 & 30.5 & - & - & - & - \\
\hline Type of refractive error & & & - & - & - & - \\
\hline Hyperopia & 24 & 40.7 & - & - & - & - \\
\hline Myopia & 20 & 33.9 & - & - & - & - \\
\hline Not detected & 15 & 25.4 & - & - & - & - \\
\hline Mean refraction & - & - & - & - & $-0.17 \pm 2.44$ & - \\
\hline Range (D) & - & - & - & - & - & -6.00 to 4.75 \\
\hline Mean corneal power & - & - & - & - & $43.60 \pm 1.96$ & - \\
\hline Range (D) & - & - & - & - & - & 39.63 to 48.50 \\
\hline Mean axial length of the eye & - & - & - & - & $23.61 \pm 1.17$ & - \\
\hline Mean uncorrected VA & - & - & - & - & $0.13 \pm 0.17$ & - \\
\hline Range & - & - & - & - & - & 0.002 to 0.60 \\
\hline Mean best-corrected VA & - & - & - & - & $0.17 \pm 0.20$ & - \\
\hline Range & - & - & - & - & - & 0.002 to 0.80 \\
\hline
\end{tabular}

D, dioptre; K, keratometry; VA, visual acuity; s.d., standard deviation.

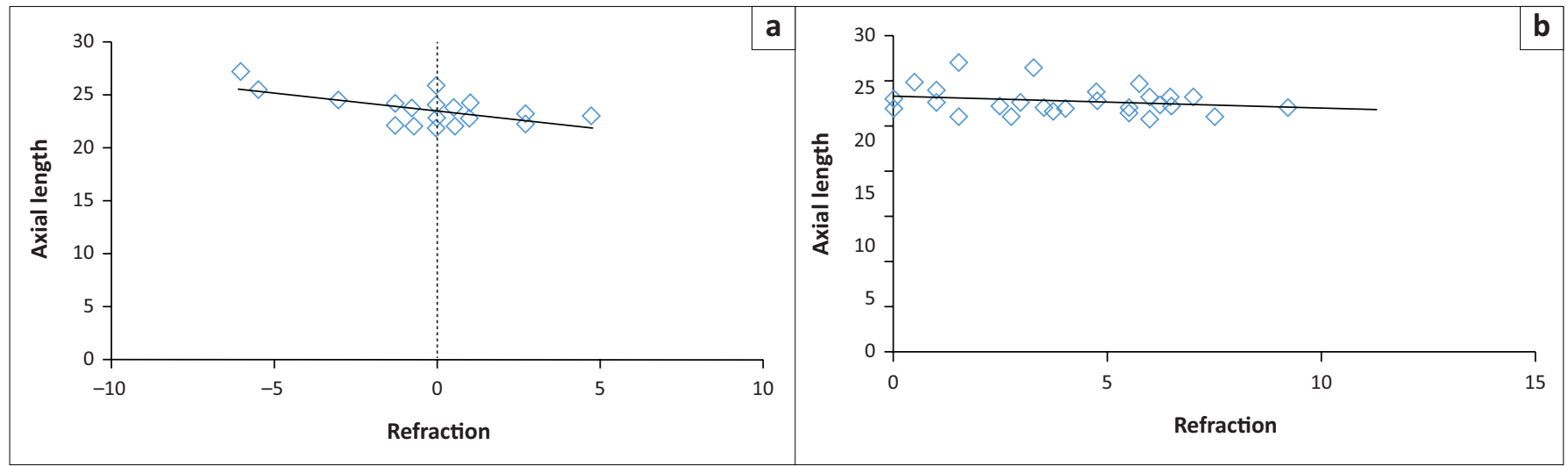

FIGURE 1: Correlation between the refractive status and axial length of the eye (a) pre-surgery and (b) post-surgery. 


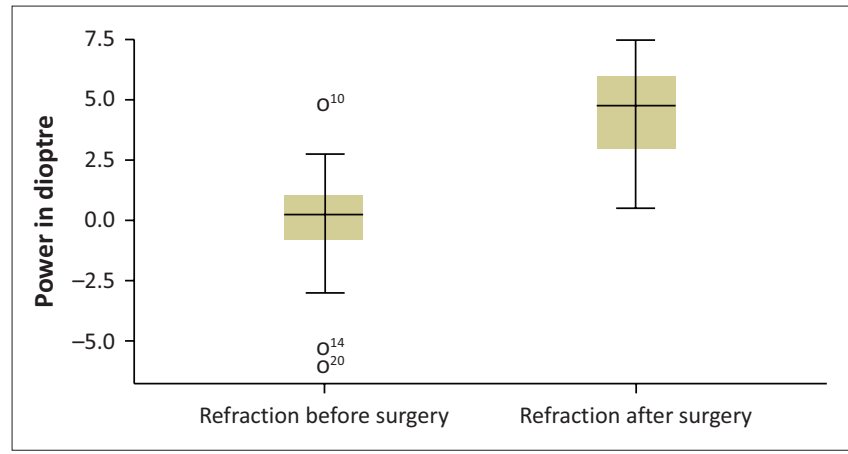

FIGURE 2: Refraction before and after surgery.

several studies published previously, ${ }^{5,8,9,15,16}$ in which authors found refraction after SOT in phakic eyes was shifted towards hypermetropia. Moreover, a comparable result was found by $\mathrm{Seo}^{13}$ and his colleagues who described that the change in refractive error varies with percentages of the vitreous cavity filled by $\mathrm{SO}$ and the state of the lens. They observed a mean change in the refraction of $+6.70 \mathrm{D}$ in phakic eyes and $-6.70 \mathrm{D}$ in aphakic eyes. However, in pseudophakic eyes, the change is variable depending on the types of intraocular lens used. ${ }^{13,17}$ The hypermetropic shift after injection of SO into the eye was attributed to the higher refractive index of silicon (1.405) compared with the vitreous (1.336). ${ }^{17,19,20}$

The corneal power for the studied group was found to be increased slightly after the operation, However, this increment did not have a clear effect in refraction after the operation $\left(R^{2}=0.011 ; p=0.597\right.$ : linear regression test). Thus, in this study, several aspects as mentioned above may play a role in the shift in refraction; however, the change in the corneal power post-surgery may not be a part of these factors. These findings were comparable with those of the preceding studies. ${ }^{5,8,9,15,16}$

The current study showed a significant change in overall axial length; a longer axial length mean was reported post-operative $(p<0.001)$ compared with the baseline measurements. Similar findings were described in previous studies $^{15,17}$ that reported a significant increment in axial length after injection of SO following PPV. Seo et al. ${ }^{14}$ observed that the anterior surface of the SO and convexity of the silicon sphere might act as a hypermetropic lens and affect the axial length and could lead to a change in refraction. From a refractive point of view, elongation of axial length induces a myopic shift in refraction as reported in several studies. . $12,22,23,24,25,26,27,28$ The post-operative elongation of the axial length of the eye results in a myopic effect. However, the findings of the current study revealed a significant hypermetropic shift. Consequently, the change in corneal curvature and axial length did not explain the hypermetropic shift after SOT in PPV. It would be interesting for future studies to assess change in refraction, axial length, keratometry and ACD pre- and post-operatively in a prospective randomised study. This is the first study conducted amongst the Sudanese population to evaluate refractive state changes following SOT in PPV.
Limitations of this study include the small sample size and its retrospective nature. Despite these limitations, the study was able to provide valuable information on the biometric and refractive changes after SOT in PPV. A prospective randomised future study with measurement of axial length, keratometry, ACD, and refractive state pre- and postoperatively in larger numbers of phakic eyes will be needed.

\section{Conclusion}

A hypermetropic shift in refraction, longer axial length and non-considerable increase in corneal curvature were observed amongst Sudanese phakic patients after PPV with instillation of SO. The hypermetropic shift in phakic eyes was because of the high refractive index of SO compared with vitreous rather than the changes in ocular biometry.

\section{Acknowledgements}

The authors are grateful to the staff of Sudan Eye Center, Khartoum who helped in data collection.

\section{Competing interests}

The authors declare that they have no financial or personal relationships that may have inappropriately influenced them in writing this article.

\section{Authors' contributions}

A.E.H., A.E.M.E. and N.M.F.A. designed the model and the computational framework and analysed the data. M.A.A. and S.H.A. carried out the implementation. M.A. performed the calculations. A.E.H. and A.E.M.E. wrote the manuscript with input from all authors. S.H.A. and M.A.A. conceived the study and were in charge of overall direction and planning.

\section{Funding information}

This research received no specific grant from any funding agency in the public, commercial or not-for-profit sectors.

\section{Data availability}

Data sharing is not applicable to this article.

\section{Disclaimer}

The views and opinions expressed in this article are those of the authors and do not necessarily reflect the official policy or position of any affiliated agency of the authors.

\section{References}

1. Barca F, Caporossi T, Rizzo S. Silicone oil: Different physical proprieties and clinical applications. BioMed Res Int. 2014 Jan 1;2014:1-7. https://doi.org/10.1155/ 2014/502143

2. Federman JL, Schubert HD. Complications associated with the use of silicone oil in 150 eyes after retina-vitreous surgery. Ophthalmology. $1988 \mathrm{Jul}$ 1;95(7):870-876. https://doi.org/10.1016/S0161-6420(88)33080-0

3. Rahman R, Bong CX, Stephenson J. Accuracy of intraocular lens power estimation in eyes having phacovitrectomy for rhegmatogenous retinal detachment. Retina. 2014 Jul 1;34(7):1415-1420. https://doi.org/10.1097/IAE.0000000000000072 
4. Emeraro F, Morescalchi F, Russo A, Romano MR, Costagliola C. Tamponade, or filling effect: Changes of forces in myopic eyes. BioMed Res Int. 2014 Jan 1;2014:1-7. https://doi.org/10.1155/2014/618382

5. Sheen-Ophir S, Rosner M, Rubowitz A. Feasibility of using experimental high viscosity silicone oils: A pilot study. Int J Retina Vitreous. 2018 Dec;4(1):1-5. https://doi.org/10.1186/s40942-017-0105-8

6. Tranos PG, Allan B, Balidis $M$, et al. Comparison of postoperative refractive outcome in eyes undergoing combined phacovitrectomy vs cataract surgery following vitrectomy. Graefes Archiv Clin Exp Ophthalmol. 2020 Jan 10;258(5):987993. https://doi.org/10.1007/s00417-019-04583-w

7. Pavlović $S$, Tomić $Z$, Latinović $S$. Changes in ocular refraction after tamponade with silicone oil. Med Pregl. 1996 Jan 1;49(5-6):181-183. PMID: 8692092

8. Fang W, Li J, Jin X, Zhai J, Dai Y, Li Y. Refractive shift of silicone oil tamponade in pseudophakic eye. BMC Ophthalmol. 2016 Dec;16(1):1-5. https://doi. org/10.1186/s12886-016-0243-z

9. Song WK, Kim SS, Kim SE, Lee CS, Byeon SH, Kwon OW. Refractive changes in silicone oil-filled pseudophakic eyes. Investig Ophthalmol Vis Sci. 2009 Apr 28;50(13):4452.

10. Hoffman A, Wolfer J, Occelli L, et al. Refractive state following retinal reattachment and silicone oil tamponade in dogs. Am J Vet Res. 2012;73(8):1299-1304. https:// doi.org/10.2460/ajvr.73.8.1299

11. Gopal S, Lakshmi P. Refractive changes after retinal surgery. Kerala J Ophthalmol. 2011;111(2):113-114.

12. Gao $Q$, Chen $X, G e J$, et al. Refractive shifts in four selected artificial vitreous substitutes based on Gullstrand-Emsley and Liou-Brennan schematic eyes. Investig Ophthalmol Vis Sci. 2009;50:3529-3534. https://doi.org/10.1167/iovs.08-2802

13. De Silva DJ, Lim KS, Schulenburg WE. An experimental study on the effect of encircling band procedure on silicone oil emulsification. Br J Ophthalmol. 2005 Oct 1;89(10):1348-1350. https://doi.org/10.1136/bjo.2004.063768

14. Seo MS, Lim ST, Kim HD, Park BI. Changes in refraction and axial length according to the viscosity of intraocular silicone oil. Korean J Ophthalmol. 1999 Jun 1;13(1):25-29. https://doi.org/10.3341/kjo.1999.13.1.25

15. Ellakwa AF, Youssef AM. Axial length variation with the use of silicone oil tamponade. Menoufia Med J. 2020 Apr 1;33(2):617-622. https://doi.org/10.4103/ mmj.mmj_343_19

16. Mazhar-ul-Hassan AK, Qidwal U, ur Rehman A, Bhatti N. Assessment of the complications secondary to silicone oil injection after pars plana vitrectomy in rhegmatogenous retinal detachment in early post-operative phase. Pak Ophthalmol. 2011 Jun 30;27(2). https://doi.org/10.36351/pjo.v27i2.497
17. Stefansson E, Anderson Jr MM, Landers 3rd MB, Tiedeman JS, McCuen 2nd BW. Refractive changes from use of silicone oil in vitreous surgery. Retina. 1988 Jan 1;8(1):20-23. https://doi.org/10.1097/00006982-198808010-00005

18. Grinbaum A, Treister G, Moisseiev J. Predicted and actual refraction after intraocular lens implantation in eyes with silicone oil. J Cataract Refractive Surg. 1996 Jul 1;22(6):726-729. https://doi.org/10.1016/S0886-3350(96)80311-7

19. Othman RN, Kamaruddin Z, Vengadasalam SR, Mustapha M. Ocular biometry and refractive changes post sutureless vitrectomy surgery [homepage on the Internet]. 2019 [cited 2021 Feb 12]; p. 114. Available from: www.myjo.org

20. Wang JK, Chang SW. Refractive results of phacoemulsification in vitrectomized patients. Int Ophthalmol. 2017 Jun;37(3):673-681. https://doi.org/10.1007/ s10792-016-0325-1

21. Liu YL, Yang CM, Huang JY, Chen MS, Hou YC. Postoperative hyperopic shift after cataract surgery in silicone oil-filled eyes. Acta Ophthalmol. 2014 Jun;92(4):e334-e335. https://doi.org/10.1111/aos.12264

22. Elmadina AM. The axial length and corneal radius of curvature ratio in Sudanese adult myopes. J Niger Optom Assoc. 2019 Aug 28;21(1):43-48.

23. Elrahman NF, Elmadina AM, Qureshi MA, Younis AM, Ahmad MI, Zain MMM Adult myopes; Relationship of central corneal thicknesses (CCT), keratometry mean (KM) and axial length (AL) among Sudanese adult myopes. Prof Med J. 2018;25(11):1660-1666. https://doi.org/10.29309/TPMJ/18.4855

24. Badmus SA, Ajaiyeoba Al, Adegbehingbe BO, Onakpoya OH, Adeoye AO. Axia length/corneal radius of curvature ratio and refractive status in an adult Nigerian population. Niger J Clin Pract. 2017;20(10):1328-1334. https://doi.org/10.4103/ njcp.njcp_183_16

25. He X, Zou H, Lu L, et al. Axial length/corneal radius ratio: Association with refractive state and role on myopia detection combined with visual acuity in Chinese schoolchildren. PLoS One. 2015 Feb 18;10(2):e0111766. https://doi. org/10.1371/journal.pone.0111766

26. Yin $G$, Wang $Y X$, Zheng $Z Y$, et al. Ocular axial length and its associations in Chinese: The Beijing Eye Study. PLoS One. 2012 Aug 21;7(8):e43172. https://doi. org/10.1371/journal.pone.0043172

27. Bhardwaj V, Rajeshbhai GP. Axial length, anterior chamber depth-a study in different age groups and refractive errors. J Clin Diagn Res. 2013 Oct;7(10):2211. https://doi.org/10.7860/JCDR/2013/7015.3473

28. Meng W, Butterworth J, Malecaze F, Calvas P. Axial length of myopia: A review of current research. Ophthalmologica. 2011;225(3):127-134. https://doi.org/10.1159/ 000317072 